\title{
A man with an itchy auricular mass lesion
}

Yang Ho Lee, Kang Duk Suh, Seog-Kyun Mun

\section{CASE}

A man aged 47 years presented with a sixmonth history of an itchy and enlarging mass on his left auricle. The patient remembered that the itching sensation first began about six months earlier and the mass had enlarged gradually over the last two months. He did not recall any trauma or piercing history at the site prior to the onset of the lesion. The patient had no past medical history, had never been exposed to toxic substances and had not experienced any excessive sun exposures. On physical examination, there was a $2 \times 1.5 \mathrm{~cm}$ flesh-coloured hard mass with a smooth surface and central pore on the antihelix of the patient's left auricle (Figure 1). In order to confirm the diagnosis, skin biopsy was done. Numerous small follicles radiating from

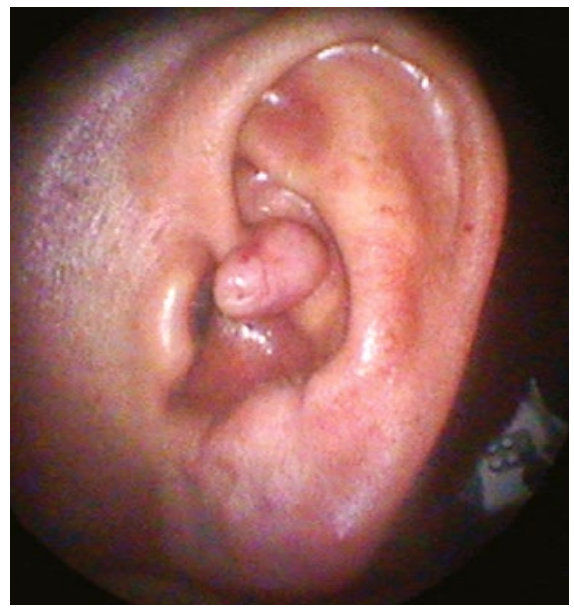

Figure 1. A solitary $1.5 \mathrm{~cm}$ well-demarcated skin-coloured mass in the left auricle a large cystic follicle lined by squamous epithelium were found in the biopsy specimen (Figure 2). The follicles were surrounded by a well-circumscribed dense stroma. The secondary follicles showed various stages of differentiation, and an inner and outer root sheath was seen. In the inner root sheath, a darkly staining eosinophilic trichohyaline granule was observed (Figure 3 ).

\section{Question 1}

What is the most likely diagnosis?

\section{Question 2}

How does the diagnosed condition typically present?

\section{Question 3}

Which demographic does this condition usually affect?

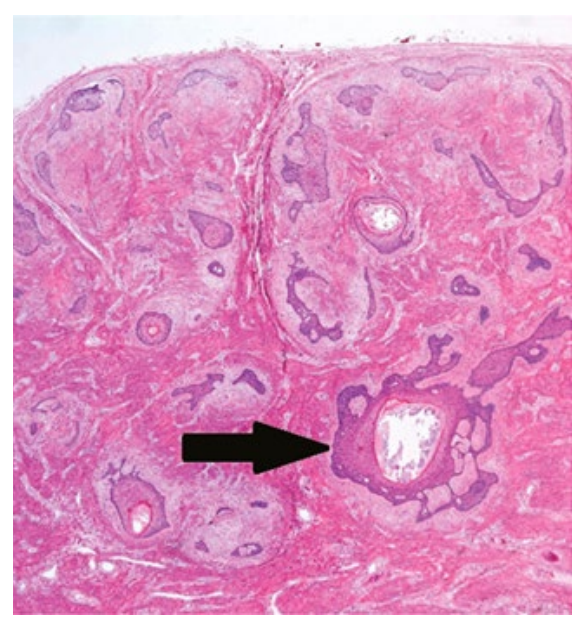

Figure 2. The central dilated primary hair follicle is filled with keratin, and secondary hair follicles are radiating from this primary hair follicle (H\&E, x40)

\section{Question 4}

What other differential diagnoses should be considered in this case?

\section{Question 5}

What diagnostic tests need to be considered to confirm the diagnosis?

\section{Question 6}

What treatment is required for this diagnosis?

\section{Answer 1}

This case shows characteristics of trichofolliculoma, a rare adnexal tumour that shows follicular differentiation and occurs solitarily on the face or scalp. It is usually considered to be a benign tumour, but there is one report of perineural invasion of trichofolliculoma. ${ }^{1}$ All stages of follicular differentiation may be seen

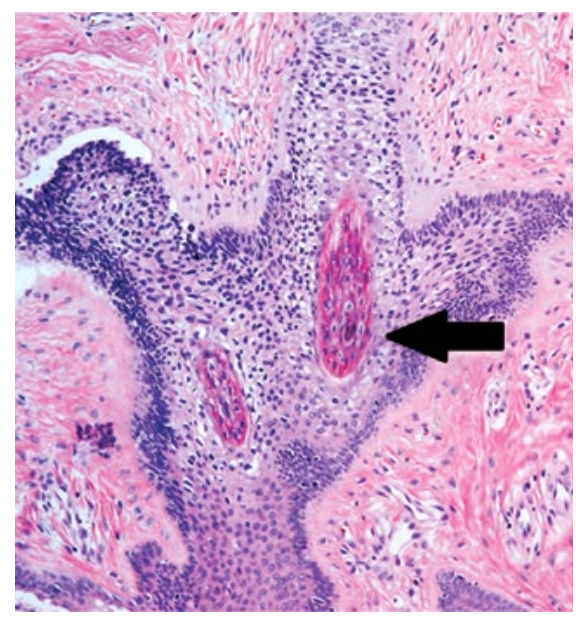

Figure 3. The secondary hair follicles show an outer and inner root sheath with eosinophilic trichohyaline granules $(H \& E, \times 100)$ 
in the lesion, and it shows differentiation of middle stage trichoepithelioma, in which the hair follicle nevus and follicular maturation are not seen. ${ }^{2}$

\section{Answer 2}

In clinical findings, trichofolliculoma is variable in size, with a hard, domeshaped, skin-coloured papule. In typical trichofolliculoma lesions, a central pore with a whitish immature hair is observed, which has diagnostic value. ${ }^{3}$ In some cases, sebum or similar materials are secreted from the central pore. There are generally no subjective or systemic symptoms, but the patient in this case complained of an itching sensation. ${ }^{4}$ This symptom is probably caused by continuous stimulation to the skin lesion caused by the loss of immature hair.

\section{Answer 3}

Trichofolliculoma may occur at any age but typically affects adults. ${ }^{1}$

\section{Answer 4}

As there were no significant findings involving skin problems in other locations, differential diagnoses should include the following.

\section{- Epidermal cyst}

These cysts are elastic but firm, with a surface colour of normal skin or a light blue and a black punctate opening at the centre. They are asymptomatic. When pressed after excision, the cyst exudes a putrid-smelling, white, gruel-like discharge, which was not seen in this particular case. Pathologically, the wall of the cyst has the same structure as normal epidermis. However, instead of the horny cell layer, there are gruel-like, layered keratinous contents. ${ }^{5}$

\section{- Soft fibroma}

The primary condition of soft fibroma is proliferation of collagen bundles with few fibroblasts. In soft fibroma, fat cells are contained in tumours in many cases. ${ }^{5}$ There were no collagen bundles with fibroblasts in this case.

\section{- Keloid}

The clinical features of keloid are a flat, sharply margined, red or brown elevation caused by proliferation of connective tissue following an injury. ${ }^{5}$ No pre-existing scar was present in this patient.

\section{- Chondroma}

Histologically, a well-circumscribed tumour with lobules of mature chondrocytes or chondroblasts surrounded by hyaline matrix is seen. ${ }^{6}$

\section{- Dilated pore of Winer}

This condition is similar to trichofolliculoma in that they both contain cystic spaces filled with keratin and are formed by squamous epithelium formed from the epidermis. However, in a dilated pore of Winer, proliferation of the follicle epithelium into adjacent tissue is less severe and shows finger-shaped proliferation. ${ }^{2}$

\section{- Pilar sheath acanthoma}

This condition also shows cystic spaces filled with keratin and squamous epithelium from epidermis, but it shows a low level of differentiation. Hair shaft in the cyst is not seen, and it rarely involves fibrovascular proliferation of adjacent tissues. $^{3}$

\section{Answer 5}

Biopsy should be considered to confirm the diagnosis and exclude other conditions such as epidermal cyst, soft fibroma, chondroma.

\section{Answer 6}

Simple excision is recommended for treatment, although there have been reports of recurrence after surgery. ${ }^{7}$ However, as there have been reports of tumour cell invasion into surrounding nerves, histological confirmation must be made. In cases where invasion has been confirmed, radical excision must be considered. ${ }^{8}$

\section{Authors}

Yang Ho Lee MD, Department of Otorhinolaryngology - Head and Neck Surgery, College of Medicine, Chung-Ang University, Seoul, Korea
Kang Duk Suh MD, Department of

Otorhinolaryngology - Head and Neck Surgery, College of Medicine, Chung-Ang University, Seoul, Korea

Seog-Kyun Mun MD, PhD, Department of Otorhinolaryngology - Head and Neck Surgery, College of Medicine, Chung-Ang University, Seoul, Korea.entdoctor@cau.ac.kr

Competing interests: None.

Funding: None.

Provenance and peer review: Not commissioned, externally peer reviewed.

\section{References}

1. Mizutani H, Senga K, Ueda M. Trichofolliculoma of the upper lip: Report of a case. Int J Oral Maxillofac Surg 1999;28(2):135-36.

2. Wu YH. Folliculosebaceous cystic hamartoma or trichofolliculoma? A spectrum of hamartomatous changes inducted by perifollicular stroma in the follicular epithelium. J Cutan Pathol 2008;35(9):843-48. doi: 10.1111/j.16000560.2007.00914.x.

3. Misago N, Kimura T, Toda S, Mori T, Narisawa Y. A revaluation of trichofolliculoma: The histopathological and immunohistochemical features. Am J Dermatopathol 2010;32(1):35-43. doi: 10.1097/DAD.0b013e3181a77414.

4. Lim P, Kossard S. Trichofolliculoma with mucinosis. Am J Dermatopathol 2009;31(4):405-06. doi: 10.1097/DAD.0b013e3181a09960.

5. Shimizu H. Benign skin tumors. In: Shimizu's textbook of dermatology. Japan: Department of Dermatology Hokkaido University, 2006; p. 439-80.

6. Podder D, Monappa V, Shetty P. Soft tissue chondroma: A rare tumor presenting as a cutaneous nodule. New Zealand: Our Dermatology Online/Nasza Dermatologia Online, 2015.

7. Srivastava RN, Ajwani KD. Trichofolliculoma. Ear Nose Throat J 1979;58(4):159-60.

8. O'Mahony JJ. Trichofolliculoma of the external auditory meatus. Report of a case and a review of the literature. J Laryngol Otol 1981;95(6):623-25. 